\title{
A Methodology to Identify Consensus Classes from Clustering Algorithms Applied to Immunohistochemical Data from Breast Cancer Patients
}

\author{
Daniele Soria $^{\mathrm{a}}$, Jonathan M. Garibaldi ${ }^{\mathrm{a}, *}$, Federico Ambrogi ${ }^{\mathrm{c}}$, Andrew R. Green ${ }^{\mathrm{b}}$, Des Powe ${ }^{\mathrm{b}}$, Emad \\ Rakha $^{\mathrm{b}}$, R. Douglas Macmillan ${ }^{\mathrm{f}}$, Roger W. Blamey ${ }^{\mathrm{f}}$, Graham Ball ${ }^{\mathrm{d}}$, Paulo J.G. Lisboa ${ }^{\mathrm{e}}$, Terence A. \\ Etchells $^{\mathrm{e}}$, Patrizia Boracchic ${ }^{\mathrm{c}}$, Elia Biganzolic ${ }^{\mathrm{c}}$, Ian O. Ellis ${ }^{\mathrm{b}}$ \\ ${ }^{a}$ School of Computer Science, University of Nottingham, Jubilee Campus, Wollaton Road, Nottingham, NG8 1BB, UK \\ ${ }^{b}$ School of Molecular Medical Sciences, Nottingham University Hospitals and University of Nottingham, \\ Queens Medical Centre, Derby Road, Nottingham, NG7 2UH, UK \\ ${ }^{c}$ Institute of Medical Statistics and Biometry, University of Milan, Via Venezian 1, 20133 Milan, Italy \\ ${ }^{d}$ School of Science and Technology, Nottingham Trent University, Clifton Campus, \\ Clifton Lane, Nottingham, NG11 8NS, UK \\ ${ }^{e}$ School of Computing and Mathematical Sciences, Liverpool John Moores University, Byrom Street, Liverpool, L3 3 AF, UK \\ ${ }^{f}$ The Breast Institute, Nottingham City Hospital, Hucknall Road, Nottingham, NG5 1PB, UK
}

\begin{abstract}
Single clustering methods have often been used to elucidate clusters in high dimensional medical data, even though reliance on a single algorithm is known to be problematic. In this paper, we present a methodology to determine a set of 'core classes' by using a range of techniques to reach consensus across several different clustering algorithms, and to ascertain the key characteristics of these classes. We apply the methodology to immunohistochemical data from breast cancer patients. In doing so, we identify six core classes, of which several may be novel sub-groups not previously emphasised in literature.
\end{abstract}

Key words: breast cancer, molecular classification, clustering methods, consensus clustering, validity indices

\section{Introduction}

Breast cancer, the most common cancer in women $[1,2]$, is a complex disease characterized by multiple molecular alterations. Current routine clinical management relies on availability of robust clinical and pathologic prognostic and predictive factors to support decision making. Recent advances in high-throughput molecular technologies supported the evidence of a biologic heterogeneity of breast cancer.

Following the seminal paper of Eisen and colleagues [3], in which hierarchical clustering and visual inspection of the dendrogram were performed to discover unknown pattern of gene associations, the use of clustering has become more and more popular, especially for discovering profiles in cancer with respect to high-throughput genomic data. Perou et al. [4] identified four molecular distinct breast cancer groups

\footnotetext{
${ }^{*}$ Corresponding author. School of Computer Science, University of Nottingham, Jubilee Campus, Wollaton Road, NG8 1BB, UK. Tel.: +441159514216

Email addresses: jmg@cs.nott.ac.uk (Jonathan M. Garibaldi)

Preprint submitted to Computers in Biology and Medicine

October 30, 2009
} 

positive, HER2 positive, basal-like and normal breast-like. A subsequent study extended this by dividing the luminal/ER positive group into three subtypes: luminal-A, B, and C [5], but the luminal-C group was later eliminated [6]. Sotiriou et al. [7] showed six similar groups, with two basal-like subgroups and no normal breast-like group. Whilst numerous studies have reported these and other novel molecular subtypes, and assigned a prognostic significance to the proposed classes $[8,9,10]$, they remain varied in their detailed classification [11]. An alternative approach to gene expression profiling is to use established robust laboratory technology, such as immunocytochemistry on formalin fixed paraffin embedded patient tumour samples. We and others have applied protein biomarker panels with known relevance to breast cancer, to large numbers of cases using tissue microarrays, exploring the existence and clinical significance of distinct breast cancer classes $[12,13,14,15,16,17,18,19]$. In particular, in [12] five breast cancer classes were identified and characterised. Note that a sixth group of only four cases was also identified but considered too small for further detailed assessment. However, these studies have not addressed the stability of the proposed classifications across different case sets, assay methods and data analysis procedures. Such an issue appears of critical relevance considering the increase in the number of features involved in bionformatics analyses.

In order to deal with the stability of classifications and in particular of clustering techniques, several studies have focused on the comparison and concordance among different clustering methods defining what is now known as the 'consensus clustering'. Monti and colleagues presented a new methodology of class discovery and clustering validation tailored to the task of analyzing gene expression data [20]. The new methodology, termed 'consensus clustering', provides a method, in conjunction with resampling techniques, to represent the consensus across multiple runs of a clustering algorithm and to assess the stability of the discovered clusters. The basic assumption of this method was the following: if the data represent a sample of items drawn from distinct sub-populations, and if a different sample drawn from the same sub-populations were to be observed, the induced cluster composition and number should not be radically different. Therefore, the more the attained clusters are robust to sampling variability, the more one can be confident that these clusters represent real structure.

Swift and colleagues used consensus clustering to improve confidence in gene-expression analysis, on the assumption that microarray analysis using clustering algorithms can suffer from lack of inter-method consistency in assigning related gene-expression profiles to clusters [21]. To assess gene-expression cluster consistency, the use of the weighted-kappa metric was analysed. This metric rates the agreement between the classification decisions made by two or more observers. In this case the two observers are the clustering methods.

Filkov and Skiena proposed a methodology for consensus clustering as an approach to integrating diverse sources of similarity clustered microarray data [22]. They proposed to exploit the popularity of cluster analysis of biological data by integrating clusterings from existing data sets into a single representative 
clustering based on pairwise similarities of the clusterings. Under reasonable conditions, the consensus cluster should provide additional information to that of the union of individual data analyses. The goals of consensus clustering are to integrate multiple data sets for ease of inspection, and to eliminate the likely noise and incongruencies from the original classifications. In terms of similarity the consensus partition should be close to all given ones, or in terms of distance, it must not be too far from any of them. One way to do this is to find a partition that minimises the distance to all the other partitions. So, given $k$ different partitions, the target one was identified as the consensus partition.

In another approach [23], robust clusters were identified by the implementation of a new algorithm termed 'Clusterfusion'. 'Clusterfusion' takes the results of different clustering algorithms and generates a set of robust clusters based upon the consensus of the different results of each algorithm. Firstly, an agreement matrix was generated with each cell containing the number of agreements amongst methods for clustering together the two variables represented by the indexing row and column indices. This matrix was then used to cluster variables based upon their cluster agreement. In essence, a clustering technique was applied to the clustering results.

The idea of combining and comparing the results of different clustering algorithms is particularly important in order to evaluate the stability of a proposed classification. In this paper, a methodology is presented to evaluate the stability of six breast cancer classes by comparing the clustering solutions provided by different algorithms. In order to address the standard problem of consensus clustering in which the label of classes is arbitrary, a label was assigned using the six clusters characterised in the work of Abd El-Rehim [12], as a reference for the description of our resulting groups.

\section{Material and Methods}

The four-step methodology for elucidating core, stable classes (groups) of data from a complex, multidimensional dataset was as follows:

1. A variety of clustering algorithms were run on the data set (see Section 2.1).

2. Where appropriate, the most appropriate number of clusters was investigated by means of cluster validity indices (see Section 2.2).

3. Concordance between clusters, assessed both visually and statistically, was used to guide the formation of stable 'core' classes of data.

4. A variety of methods were utilised to characterise the elucidated core classes.

The methodology was applied to a well-known set of data concerning breast cancer patients [12] (see Section 2.5) in order to obtain core classes. Once these core classes were obtained, the clinical relevance of 
the corresponding patient groups were investigated by means of associations with related patient data. All statistical analysis was done using $R$, a free software environment for statistical computing and graphics [24].

\subsection{Clustering algorithms}

Five different algorithms were used for cluster analysis:

i. Hierarchical (as per our previous study [12])

ii. K-means (KM)

iii. Partitioning around medoids (PAM)

iv. Adaptive resonance theory (ART)

v. Fuzzy c-means (FCM)

\subsubsection{Hierarchical clustering}

The hierarchical clustering algorithm (HCA) begins with all data considered to be in a separate cluster. It then finds the pair of data with the minimum value of some specified distance metric; this pair is then assigned to one cluster. The process continues iteratively until all data are in the same (one) cluster. A conventional hierarchical clustering algorithm (HCA) was utilised, utilising Euclidean distance on the raw (unnormalised) data with all attributes equally weighted.

\subsubsection{K-means clustering}

The K-means (KM) technique aims to partition the data into $K$ clusters such that the sum of squares from points to the assigned cluster centres is minimised. The algorithm repeatedly moves all cluster centres to the mean of their Voronoi sets (the set of data points which are nearest to the cluster centre). The objective function minimised is:

$$
J(V)=\sum_{j=1}^{k} \sum_{i=1}^{c_{j}}\left\|x_{i}-v_{j}\right\|^{2}
$$

where $x_{i}$ is the $i$-th datum, $v_{j}$ is the $j$-th cluster centre, $k$ is the number of clusters, $c_{j}$ is the number of data points in the cluster $j$ and $\left\|x_{i}-v_{j}\right\|$ is the Euclidean distance between $x_{i}$ and $v_{j}$.

The $j$-th centre $v_{j}$ can be calculated as:

$$
v_{j}=\frac{1}{c_{j}} \sum_{i=1}^{c_{j}} x_{i}, \quad j=1, \ldots, k .
$$

K-means clustering is dependent on the initial cluster centres setting (which, in turn, determines the initial cluster assignment). Various techniques have been proposed for the initialisation of clusters [25], but for this study we used a fixed initialisation of the cluster centres obtained with hierarchical clustering. The number of clusters is an explicit input parameter to the K-means algorithm. 


\subsubsection{Partitioning around medoids}

The partitioning around medoids (PAM) algorithm (also known as the $k$-medoids algorithm) is a technique which attempts to minimise the distance between points labelled to be in a cluster and a point designated as the centre of that cluster. In contrast to the K-means algorithm, PAM chooses data points as centres (the so-called medoids) and then assigns each point to its nearest medoid. A medoid is defined as the object within a cluster for which the average dissimilarity to all other objects in the cluster is minimal, i.e. it is the most centrally located datum in the given cluster. Dissimilarities are nonnegative numbers that are small (close to zero) when two data points are 'near' to each other and become large when the points are very different [26]. Usually, a Euclidean metric is used for calculating dissimilarities between observations.

The algorithm consists of two phases: the build phase in which an initial set of $k$ representative medoids is selected and the swap phase in which a search is carried out to improve the choice of medoids (and hence the cluster allocations). The algorithm is described in detail in [26], pp.102-104. The number of clusters is an explicit input parameter to the PAM algorithm.

\subsubsection{Adaptive resonance theory}

The adaptive resonance theory (ART) algorithm has three main steps [27]. First, the data are normalised to a unit hypersphere, thus representing only the ratios between the various dimensions of the data. Second, data allocated to each cluster are required to be within a fixed maximum solid angle of the group mean, controlled by a so-called 'vigilance parameter' $\rho$, namely $X_{k} \cdot P^{i} \leq \rho$. However, even when the observation profile and a prototype are closer than the maximum aperture for the group, a further test is applied to ensure that the profile and prototype have the same dominant covariates. This is done in a third step by specifying the extent to which the nearest permissible prototype allocation for the given observation must be on the same side of the data space from the diagonal comprising a vector of ones, $\hat{1}$, using a pre-set parameter, $\lambda$ :

$$
X_{k} \cdot P^{i} \leq \lambda X_{k} \cdot \hat{1}
$$

The ART algorithm is initialised with no prototypes and creates them during each successive pass over the data set. It has some, limited, sensitivity to the order in which the data are presented and converges in a few iterations. In the ART algorithm the clusters are determined automatically: the number of clusters is not an explicit parameter, although there are parameters that can adjust the number obtained.

\subsubsection{Fuzzy c-means}

The fuzzy c-means (FCM) algorithm is a generalisation of the K-means algorithm which is based on the idea of permitting each object to be a member of every cluster to a certain degree, rather than an object having to belong to only one cluster at any one time. It is based upon the concept of fuzzy logic promulgated 
by Zadeh [28] and aims to minimise the objective function

$$
J(U, V)=\sum_{i=1}^{n} \sum_{j=1}^{c}\left(\mu_{i, j}\right)^{m}\left\|x_{i}-v_{j}\right\|^{2}
$$

where $n$ is the number of data points, $x_{i}$ and $v_{j}$ are the data points and cluster centres and $\mu_{i, j}$ is the membership degree of data $x_{i}$ to the cluster centre $v_{j}\left(\mu_{i, j} \in[0,1]\right) . m$ is called the 'fuzziness index' and the value of $m=2.0$ is usually chosen. An exhaustive description of this method can be found in [29]. As for $\mathrm{K}$-means, the number of clusters is an explicit input parameter to FCM.

\subsection{Cluster validity}

Clustering validity is a concept that is used to evaluate the quality of clustering results. If the number of clusters is not known prior to commencing an algorithm, a cluster validity index may be used to determine the best number of clusters for the given data set. Although there are many variations of validity indices, they are all either based on considering the data dispersion in a cluster and between clusters, or considering the scatter matrix of the data points and the one of the clusters centers. In this study, the following indices were applied to those algorithms for which the number of clusters is an explicit parameter, over a range of number of clusters:

1. Calinski and Harabasz [30]

2. Hartigan [31]

3. Scott and Symons [32]

4. Marriot [33]

5. $\operatorname{Trace} W[34,35]$

6. $\operatorname{Trace} W^{-1} B[35]$

For each index, the number of clusters to be considered was chosen according to the rule reported in Table 1 where $i_{n}$ is the validity index value obtained for $n$ clusters [36].

[Table 1 about here.]

\subsection{Derivation of classes}

Concordance among solutions was evaluated using the Cohen's kappa coefficient $\kappa$ [37]. This coefficient is a statistical measure of inter-rater agreement for qualitative (categorical) items. It is generally thought to be a more robust measure than simple percent agreement calculation since $\kappa$ takes into account the agreement occurring by chance. 
To enable cluster visualisation, the original data space (consisting of a large number of dimensions) was transformed by principal component analysis (PCA) [38], and then the points plotted at their projected position on axes of the first and second principal components. As PCA transforms data such that the first principal component $(\mathrm{PC})$ carries the maximum amount of variance in the data and the second $\mathrm{PC}$ carries the next largest variance (etc.), such a plot provides a picture in which the clusters have been 'spread out' as much as possible.

The previously obtained clustering results from Abd El-Rehim and colleagues [12], the cluster validity indices (where appropriate), visualisation of the new clustering results themselves, and the concordance among clustering solutions were then all used heuristically to guide the formulation of a set of rules to define core class membership from the various cluster assignments.

\subsection{Characterisation of classes}

\subsubsection{Class characterisation by visualisation}

For inspection of the patient characteristics in each class, the distribution of each variable in the class was compared with its distribution in the total sample, using boxplots. A boxplot shows the median expression level (solid horizontal bar), the upper quartile and lower quartile range (shaded grey bar), the highest nonoutlier and lowest non-outlier (smaller ticks joined by dashed lines), and any outliers (open circles). For a full description of boxplots, including the statistical definition of outliers see, for example, [39].

\subsubsection{Class characterisation by OSRE (orthogonal search rule extraction)}

Orthogonal Search Rule Extraction (OSRE) [40] is a computationally efficient algorithm to search for hypercubes in data space, since they map directly onto Boolean rules. A general description of this method is given below, while a more detailed one can be found in [40]. This methodology initially returns a rule for each data point, which triggers a pruning process to keep only those rules which represent large proportions of the data in the clusters, and do so with minimal mixing between clusters. The result is a set of low-order rules containing the covariates that characterize the sub-group of the cluster. The proposed interpretation is that these rules identify the drivers for cluster allocation, which may vary across the cluster but are, in general, well-defined.

Note that this method contrasts with widely used rule induction methods in two ways: firstly, there are no univariate cut-offs for groups of data, as in OSRE a sequential univariate search is carried out at the level of each individual data point which returns a multivariate hyperbox around that point, without the need to partition the data along a sequence of univariate covariates; and secondly, that the rules are overlapping, rather than constrained to mutual exclusivity as is usually the case in rule tree induction. 


\subsubsection{Class characterisation by ANN (Artificial Neural Networks)}

A conventional multi-layer perceptron artificial neural network (MLP-ANN) model was utilised such that individual H-scores derived from the tissue microarray analysis of the clinical samples were set as inputs and the class was set as the output using Boolean notation (i.e. 1 represented membership of a given class, 0 represented non-membership). This allowed the identification of markers that drive membership of a given class and that discriminate the class from the others. A three-layer MLP-ANN (featuring eight nodes in the hidden layer) with a back-propagation algorithm and a sigmoid activation function was used. The approach used in this work is similar to the ones used in [41] and [42].

\subsection{Patients and clinical methods}

A series of 1076 patients from the Nottingham Tenovus Primary Breast Carcinoma Series presenting with primary operable (stages I, II and III) invasive breast cancer between 1986-98 was used to evaluate the methodology. Immunohistochemical reactivity for twenty-five proteins, with known relevance in breast cancer including those used in routine clinical practice, were previously determined using standard immunocytochemical techniques on tumour samples prepared as tissue microarrays [12]. Levels of immunohistochemical reactivity were determined by microscopical analysis using the modified H-score (values between 0-300), giving a semiquantitative assessment of both the intensity of staining and the percentage of positive cells. For the intensity, a score of 0 to 3, corresponding to negative, weak, moderate and strong positivity, was recorded. In addition, the percentage of positive cells at each intensity category was estimated. The H-score is calculated as follows [43]:

$$
\begin{aligned}
\text { H-score }= & (1 \times \% \text { of cells stained at intensisty category } 1) \\
& +(2 \times \% \text { of cells stained at intensisty category } 2) \\
& +(3 \times \% \text { of cells stained at intensisty category } 3)
\end{aligned}
$$

The range of possible scores is thus 0 to 300, where 300 equals $100 \%$ of tumour cells stained strongly [44]. The complete list of variables used in this study is given in Table 2, while data extracted from three patients is reported as an example in Table 3.

[Table 2 about here.]

[Table 3 about here.]

This is a well-characterised series [12] of patients who were treated according to standard clinical protocols. Patient management was based on tumour characteristics using Nottingham Prognostic Index (NPI) and hormone receptor status. Patients with an NPI score $\leq 3.4$ received no adjuvant therapy, those with a NPI score $>3.4$ received hormone therapy if oestrogen receptor (ER) positive or classical cyclophosphamide, 
methotrexate and 5-fluorouracil (CMF) if ER negative and fit enough to tolerate chemotherapy. Hormonal therapy was given to 420 patients (39\%) and chemotherapy to 264 (24.5\%). This study was approved by the Nottingham Research Ethics Committee 2 under the title 'Development of a molecular genetic classification of breast cancer'.

\section{Results}

\subsection{Clustering results}

\subsection{1. $H C A, K$-means, PAM and $A R T$}

The HCA results from Abd El-Rehim et al. [12] were utilised, unaltered. Both the K-means and PAM algorithms were run with the number of clusters varying from two to twenty, as the number of clusters is an explicit input parameter of the algorithms. Given that both algorithms can be sensitive to cluster initialisation and in order to obtain reproducible results, both techniques were initialised with the cluster assignments obtained by hierarchical clustering. For the ART algorithm, the parameters were set in order to obtain six clusters in order to match the number of clusters previously obtained by HCA. The best validity index obtained for repeated runs of the algorithm with 20 random initialisations was used to select the final clustering assignment.

\subsubsection{Fuzzy c-means}

The fuzzy c-means algorithm did not perform as hoped. When the number of clusters was set as two and three, it appeared that reasonable results were obtained. However, from examination of the membership function of each point assigned to these clusters, it could be seen that it was very close to either $1 / 2$ or $1 / 3$, respectively. In other words, every data point was assigned to all the clusters with the same membership. Moreover, when the number of clusters was above three, non-zero memberships were evident for only three clusters and these memberships were similar to the three cluster solution - i.e. for $n>3$, the $n=3$ cluster solution was obtained, but with $n-3$ empty clusters. These results indicated that the fuzzy c-means was not able to obtain clear cluster partitions.

The fuzziness index $m$ was altered in an attempt to improve the results obtained, but it was found that little difference in the results was observed until $m$ was close to one. Given that when $m=1$ fuzzy c-means is equivalent to K-means, this result was not useful. As there are many applications for which the fuzzy c-means technique has been successful (see, for example, [45]), these results are not easy to explain, but they may have been caused by the fact that our data contains a lot of values close to the extremes of each variable. Although the fuzzy c-means algorithm is widely used in literature, we decided to drop it from further analysis due to its poor performance on our data. 


\subsection{Cluster validity}

The values of the decision rule obtained for various values of the validity indices for both K-means and PAM, for 2 to 20 clusters are shown in Fig. 1; (a) shows the validity decision rule values obtained for K-means and (b) shows those obtained for PAM. The best number of clusters according to each validity index, for each clustering algorithm, corresponds to the either the maximum or minimum decision rule value (depending on the index), as indicated by the solid circle in Fig. 1.

[Figure 1 about here.]

It can be seen that, while there was not absolute agreement among the indices as to which was the best number of clusters for the K-means method, there is good agreement that the best number of clusters for the PAM method is four. Although the best number of clusters varies according to validity index for K-means, on further inspection, it can be seen from Fig. 1 that there is more agreement than might be immediately apparent. For example, the Scott and Symons index (which indicated that the best number of clusters was three) indicated that the second best number of clusters was six. Consequently, the indices were used to rank order the number of clusters and the minimum sum of ranks was examined. It was found that the minimum sum of ranks (a form of consensus among the indices) indicated that the overall best number of clusters was six for K-means and four for PAM. Furthermore, careful examination of Fig. 1(b) confirms that the six cluster solution for PAM is of relatively poor quality.

\subsection{Derivation of classes}

The correspondence of patients assigned in the six cluster solution for each of the methods was then examined. Cohen's kappa and weighted-kappa indices were computed to measure the degree of agreement among algorithms. For the weighted-kappa index, weights were set in decreasing order from one (perfect agreement) to zero (complete disagreement) with a 0.2 step between levels. Results are reported in Table 4 . From this table, a better agreement between K-means and hierarchical algorithms is evident compared to that between ART and hierarchical. It is also evident that the PAM six cluster solution has lower concordance with the original HCA results than either K-means or ART, and that the concordance of PAM with K-means and ART is also correspondingly lower.

[Table 4 about here.]

The cluster numbers were aligned with those obtained previously by Abd El-Rehim et al. in [12] in order to minimise differences and to aid visualisation. Biplots of the aligned clusters are shown in Fig. 2 for the six cluster solution from each algorithm. From these plots, it can be seen that the most similar results were obtained from the Hierarchical, K-means and ART. In fact, all these three methods obtain two clusters (1 \& 2) split over the left-hand side of the biplots. A third cluster (cluster 6) is evident towards the bottom 
of the biplot. Then various splits of remaining data into three clusters $(3,4 \& 5)$ can be seen. The PAM algorithm, instead, obtains three clusters $(1,2 \& 4)$ split over the left-hand side, one group is visible towards the bottom (cluster 6) and one is spread in the center of the biplot (cluster 3). PAM places all patients on the right-hand side into a single cluster (cluster 5).

[Figure 2 about here.]

The biplots further confirm that the six cluster solution obtained from the PAM algorithm was the most dissimilar among the considered techniques. Taking into account the results of validity indices analysis, the concordance analysis and the visual analysis, we decided to remove the six clusters determined by PAM from further analysis.

The cluster distributions (number of patients in each cluster) obtained for the original hierarchical clustering and those obtained for the K-means and ART methods are shown in Table 5.

[Table 5 about here.]

Focusing on these cluster correspondences, we wanted to define core classes containing the biggest possible number of patients. In a first attempt, considering agreement among the three clustering techniques (HCA, KM and ART) and looking at those patients assigned to the same group by different methods, a total of 382 patients were classified if hierarchical group 4 was considered and 463 if not. After that, for each labelled group, concordances between all pairs of methods were analysed. It was found that the sum of the number of patients assigned to the same group ranged between 459 (pairing HCA and ART) and 645 (pairing KM and ART). These results are again reflected in Table 4. Two principles were used to guide the definition of consensus classes: (i) to consider all the clustering techniques analysed and (ii) to get the highest number of patients assigned to any class. These principles conflict, in that strict application of the first principle leads to a decrease in the number of patients assigned to classes. Hence, a heuristic trade-off between the two was employed. As a result, hierarchical group 4 was omitted (being replaced by group 5), and the ART assignments were not considered in a strictly conjunctive manner. Consequently, a set of six core breast tumour classes was derived following the specific rules reported in Table 6, in which the resultant number of patients in each class is shown.

It was found that almost the $62 \%$ of data was assigned to these core classes; the remaining patients were placed into a 'not classified' (NC) group. It must be stressed that the derivation of class assignments was made on the basis of the clustering results alone (which are, obviously, based on the 25 markers only) - class assignments, although somewhat subjective, were made blind to all clinical and outcome data. It should be also noted that around a third (actually 38\%) of all patients were not assigned to any of the core classes.

[Table 6 about here.] 


\subsection{Characterisation of classes}

Biplots of the six consensus classes were produced and are shown in Fig. 3, in order to provide a visualisation of the separation of the classes.

[Figure 3 about here.]

Fig. 3(a) shows the biplot obtained for all patients, in which the cases not assigned to any class (NC) have been coloured grey. It can be seen that these fall mainly into the centre region of the biplot. Fig. 3(b) shows the biplot obtained for only patients assigned to classes $1-6$. It can be seen that the classes appear more spread out. The first axis was mainly determined, on the left, by luminal markers including luminal cytokeratins (CK18, CK7/8, CK19), hormone receptors (ER, AR, PgR), and MUC1 over-expression and, on the right, by basal cytokeratins (CK14 and CK5/6) and partly by p53 over-expression. The second axis is determined, on the top, partly by nuclear BRCA1 (nBRCA1) over-expression and, on the bottom, by HER2 and E-cad over-expression (also HER3 and HER4, although these are not shown as they overlap HER2).

Fig. 4 shows boxplots of all 25 markers, (a) for all cases, (b) for those cases assigned to classes 16 , and (c-h) for each class separately. By inspection of both the biplots and the boxplots, we derived a description of each class. For example, classes 1 and 2 are characterised by strong expression of the luminal CK markers, as well as moderate to strong MUC1 expression (as per the population). However, there is a distinct difference regarding HER3 and HER4 expression. It can also be seen that classes 4 and 5 both exhibit higher expressions of the basal CKs (CK5/6 and CK14). Triple negative patients with high p53 levels are grouped in class 4 , whereas class 5 consist of triple negative patients with low p53 levels. A summary of the class characteristics obtained by visual inspection of the boxplots is given in Table 7 .

The results obtained from the automated characterisation methods (MLP-ANN and OSRE) are reported in Table 8.

[Figure 4 about here.]

[Table 7 about here.]

[Table 8 about here.]

A proposed summary of the essential characterisations of the classes obtained is given in Fig. 5, according to the available bio-pathological knowledge. It is worth noting that class 2, labelled as Luminal-N, and the split of the basal group into two different subgroups depending on p53 levels, appear to be novel findings not previously emphasised in literature.

[Figure 5 about here.] 


\section{Clinical Evaluation}

\subsection{Patient clinical outcome}

Patient age ranged from 18 to 72 years (median 54 years). Of the available cases, 708 (66\%) cases were aged 50 years or more. At the time of diagnosis, 160 (14.9\%) tumours were histological grade 1, 343 (31.9\%) grade 2 and $572(53.2 \%)$ grade 3 . A total of $654(60.8 \%)$ patients had lymph node-negative disease and $419(38.9 \%)$ had positive lymph nodes (332 cases with between one and three positive nodes, 87 cases with four or more positive). Frequencies for histological tumour types were: 649 invasive ductal carcinomas of no special type (NST), 171 tubular mixed carcinomas, 30 medullary carcinomas, 112 lobular carcinomas, 27 tubular carcinomas, 11 mucinous carcinomas, five cribriform carcinomas, three papillary carcinomas, 37 mixed NST and lobular carcinomas, 24 mixed NST and special type carcinomas and four miscellaneous tumours. A total of $736(68.4 \%)$ had tumour size more than $1.5 \mathrm{~cm}$ and distant metastases was observed in 111 cases.

\subsection{Clinical characterisation of patients by class}

Significant associations, as expected, were found between the classes with respect to patient age, tumour grade, size, lymph node stage and histological tumour type (see Table 9).

[Table 9 about here.]

A boxplot of the Nottingham Prognostic Index (NPI) split by class is shown in Fig. 6. It can be seen that the NPI for classes 1 and 2 is lower than that of classes $3-6$ (overall Kruskal-Wallis $p<<0.001$ ). It can also be seen that classes 1 and 2 have similar NPI, and classes 3-6 have similar NPI (to each other). This is an interesting observation for two reasons. Firstly, it confirms that the NPI is providing discriminant information between classes 1 and 2, and classes 3-6. Secondly, it suggests that the class divisions are providing additional information to the NPI.

[Figure 6 about here.]

\section{Discussion}

This study has extended our previous work [12], with the application of different clustering techniques to address the issue of the non-existence of the 'perfect' clustering algorithm. In particular, in this work four different clustering methods (in addition to the hierarchical method used in [12]) were applied to a multidimensional dataset of protein biomarker data, in order to evaluate the stability of results coming from different techniques. Different clustering algorithms result in different clusters, particularly when large multi-dimensional data sets are considered. 
To explore the extent of the differences among different algorithms, an informal consensus clustering was used, grouping together patients that were assigned to 'similar' clusters by different clustering algorithms. The consensus approach was similar to the one used by Kellam et al. [23], but instead of building an agreement matrix, the previously published hierarchical clustering solution (and associated labelling) was used as a fixed reference. In this way, a set of six core classes of breast cancer was elucidated. This consensus methodology was used to combine results obtained by different clustering algorithms rather than as a comparison with previously published approaches. Another important issue that emerges when cluster analysis is performed, is the best number of clusters to consider. Several validity indices have been proposed in recent years (see, for example, [36]) to evaluate the compactness of clusters and the separation among them. For the algorithms which take an explicit number of clusters as an input parameter (i.e. K-means and PAM), cluster validity indices were used to guide the choice of the 'best' number of clusters. Note that cluster validity indices would have been applied to the fuzzy c-means algorithm had it not been dropped from analysis for the reasons outlined in Section 3.1.2.

Furthermore, this study confirmed, as already highlighted in [13], that cluster analysis should be treated with caution, as different clustering algorithms will lead to different groupings of tumours. In particular, in our case, the PAM algorithm, when run with six clusters as an input, provided groups that were different from those obtained using the other techniques. In addition, the hierarchical algorithm, commonly used in standard bioinformatics applications of cluster analysis, such as [4] or [9], seems to provide a dissimilar and skewed classification with respect to the others, thus reducing the degree of overall concordance and the number of subjects assigned to the core classes.

In conclusion, we have clearly demonstrated that different clustering algorithms can produce quite different solutions on such multi-dimensional data. It should be noted that no feature extraction was performed in this study, so avoiding a possible cause of diverse techniques not converging into similar results. We have proposed a methodology for reaching consensus from the various results that may be obtained from clustering algorithms, and have illustrated this consensus methodology on a well-known set of breast cancer data. In doing so, we have identified possible new sub-classes of breast cancer which warrant further investigation. We emphasise that this consensus methodology, by its heuristic nature, should be considered as an exploratory technique, and must not be considered as providing any form of definitive answer. Further work exploring, for example, the statistical properties of the considered algorithms may provide relevant information on the structure on this complex biological problem.

\section{Acknowledgement}

This study was, in part, funded by the Breast Cancer Campaign, and was supported by the BIOPATTERN FP6 Network of Excellence (FP6-IST-508803) and the BIOPTRAIN FP6 Marie-Curie EST Fellowship (FP6-007597). 


\section{References}

[1] D. Parkin, F. Bray, J. Ferlay, P. Pisani, Estimating the world cancer burden: Globocan 2000, Int J Cancer 94 (2001) $153-156$.

[2] F. Kamangar, G. Dores, W. Anderson, Patterns of cancer incidence, mortality, and prevalence across five continents: Defining priorities to reduce cancer disparities in different geographic regions of the world, J Clin Oncol 24 (2006) 21372150 .

[3] M. Eisen, P. Spellman, P. Brown, D. Botstein, Cluster analysis and display of genome-wide expression patterns, Proc Natl Acad Sci U S A 95 (1998) 14863-8.

[4] C. Perou, T. Sorlie, M. Eisen, M. Van De Rijn, S. Jeffrey, C. Rees, J. Pollack, D. Ross, H. Johnsen, L. Akslen, $\varnothing$. Fluge, A. Pergamenschikov, C. Williams, S. Zhu, P. Lonning, A. Børresen-Dale, P. Brown, D. Botstein, Molecular portraits of human breast tumours, Nature 406 (2000) 747-752.

[5] T. Sorlie, C. Perou, R. Tibshirani, T. Aas, S. Geisler, H. Johnsen, T. Hastie, M. Eisen, M. Van De Rijn, S. Jeffrey, T. Thorsen, H. Quist, J. Matese, P. Brown, D. Botstein, P. Eystein Lonning, A. Børresen-Dale, Gene expression patterns of breast carcinomas distinguish tumor subclasses with clinical implications, Proc Natl Acad Sci U S A 98 (2001) 1086910874.

[6] T. Sorlie, R. Tibshirani, J. Parker, T. Hastie, J. Marron, A. Nobel, S. Deng, H. Johnsen, R. Pesich, S. Geisler, J. Demeter, C. Perou, P. Lonning, P. Brown, A. Børresen-Dale, D. Botstein, Repeated observation of breast tumor subtypes in independent gene expression data sets, Proc Natl Acad Sci U S A 100 (2003) 8418-8423.

[7] C. Sotiriou, S.-Y. Neo, L. McShane, E. Korn, P. Long, A. Jazaeri, P. Martiat, S. Fox, A. Harris, E. Liu, Breast cancer classification and prognosis based on gene expression profiles from a population-based study, Proc Natl Acad Sci U S A 100 (2003) 10393-10398.

[8] S. Calza, P. Hall, G. Auer, J. Bjöhle, S. Klaar, U. Kronenwett, E. Liu, L. Miller, A. Ploner, J. Smeds, J. Bergh, Y. Pawitan, Intrinsic molecular signature of breast cancer in a population-based cohort of 412 patients, Breast Cancer Research 8:R34.

[9] L. van't Veer, H. Dai, M. van de Vijver, Y. He, A. Hart, M. Mao, H. Peterse, K. van der Kooy, M. Marton, A. Witteveen, G. Schreiber, R. Kerkhoven, C. Roberts, P. Linsley, R. Bernards, S. Friend, Gene expression profiling predicts clinical outcome of breast cancer, Nature 415 (2002) 530-536.

[10] M. West, C. Blanchette, H. Dressman, E. Huang, S. Ishida, R. Spang, H. Zuzan, J. Olson Jr., J. Marks, J. Nevins, Predicting the clinical status of human breast cancer by using gene expression profiles, Proc Natl Acad Sci U S A 98 (2001) 11462-11467.

[11] Z. Hu, C. Fan, D. Oh, J. Marron, X. He, B. Qaqish, C. Livasy, L. Carey, E. Reynolds, L. Dressler, A. Nobel, J. Parker, M. Ewend, L. Sawyer, J. Wu, Y. Liu, R. Nanda, M. Tretiakova, A. Ruiz Orrico, D. Dreher, J. Palazzo, L. Perreard, E. Nelson, M. Mone, H. Hansen, M. Mullins, J. Quackenbush, M. Ellis, O. Olopade, P. Bernard, C. Perou, The molecular portraits of breast tumors are conserved across microarray platforms, BMC Genomics 7 (2006) 96.

[12] D. Abd El-Rehim, G. Ball, S. Pinder, E. Rakha, C. Paish, J. Robertson, D. Macmillan, R. Blamey, I. Ellis, Highthroughput protein expression analysis using tissue microarray technology of a large well-characterised series identifies biologically distinct classes of breast cancer confirming recent cDNA expression analyses, Int. Journal of Cancer 116 (2005) 340-350.

[13] F. Ambrogi, E. Biganzoli, P. Querzoli, S. Ferretti, P. Boracchi, S. Alberti, E. Marubini, I. Nenci, Molecular subtyping of breast cancer from traditional tumor marker profiles using parallel clustering methods, Clinical Cancer Research 12 (3) (2006) 781-790.

[14] E. Korsching, J. Packeisen, K. Agelopoulos, M. Eisenacher, R. Voss, J. Isola, P. van Diest, B. Brandt, W. Boecker, H. Buerger, Cytogenetic alterations and cytokeratin expression patterns in breast cancer: Integrating a new model of breast differentiation into cytogenetic pathways of breast carcinogenesis, Lab Invest 82 (2002) 1525-1533. 
[15] G. Callagy, E. Cattaneo, Y. Daigo, L. Happerfield, L. Bobrow, P. Pharoah, C. Caldas, Molecular classification of breast carcinomas using tissue microarrays, Diagn Mol Pathol 12 (2003) 27-34.

[16] N. Makretsov, D. Huntsman, T. Nielsen, E. Yorida, M. Peacock, M. Cheang, S. Dunn, M. Hayes, M. van de Rijn, C. Bajdik, C. Blake Gilks, Hierarchical clustering analysis of tissue microarray immunostaining data identifies prognostically significant groups of breast carcinoma, Clin Cancer Res 10 (2004) 6143-6151.

[17] J. Jacquemier, C. Ginestier, J. Rougemont, V.-J. Bardou, E. Charafe-Jauffret, J. Geneix, J. Adélaïde, A. Koki, G. Houvenaeghel, J. Hassoun, D. Maraninchi, P. Viens, D. Birnbaum, F. Bertucci, Protein expression profiling identifies subclasses of breast cancer and predicts prognosis, Cancer Res 65 (2005) 767-779.

[18] R. Diallo-Danebrock, E. Ting, O. Gluz, A. Herr, S. Mohrmann, H. Geddert, A. Rody, K. Schaefer, S. Baldus, A. Hartmann, P. Wild, M. Burson, H. Gabbert, U. Nitz, C. Poremba, Protein expression profiling in high-risk breast cancer patients treated with high-dose or conventional dose-dense chemotherapy, Clin Cancer Res 13 (2007) 488-497.

[19] M. Dolled-Filhart, L. Rydén, M. Cregger, K. Jirström, M. Harigopal, R. Camp, D. Rimm, Classification of breast cancer using genetic algorithms and tissue microarrays, Clin Cancer Res 12 (2006) 6459-6468.

[20] S. Monti, P. Tamayo, J. Mesirov, T. Golub, Consensus clustering: A resampling-based method for class discovery and visualization of gene expression microarray data, Machine Learning 52 (2003) 91-118.

[21] S. Swift, A. Tucker, V. Vinciotti, N. Martin, C. Orengo, X. Liu, P. Kellam, Consensus clustering and functional interpretation of gene-expression data, Genome Biology 5:R94.

[22] V. Filkov, S. Skiena, Integrating microarray data by consensus clustering, in: Proceedings of the 15th IEEE International Conference on Tools with Artificial Intelligence, 2003, pp. 418-426.

[23] P. Kellam, X. Liu, N. Martin, C. Orengo, S. Swift, A. Tucker, Comparing, contrasting and combining clusters in viral gene expression data, in: Proceedings of 6th Workshop on Intelligent Data Analysis in Medicine, 2001.

[24] J. Maindonald, W. Braun, Data Analysis and Graphics Using R - An Example-Based Approach, Cambridge University Press, 2003.

[25] M. Al-Daoud, S. Roberts, New methods for the initialisation of clusters, Pattern Recognition Letters 17 (5) (1996) $451-455$.

[26] L. Kaufman, P. Rousseeuw, Finding Groups in Data: an Introduction to Cluster Analysis, Wiley series in probability and mathematical statistics. Applied Probability and Statistics. New York: Wiley, 1990.

[27] G. Carpenter, S. Grossberg, ART2: Stable self-organization of pattern recognition codes for analogue input patterns, Applied Optics 26 (1987) 4919-4930.

[28] L. Zadeh, Fuzzy sets, Inf. and Cont. 8 (1965) 338-353.

[29] J. Bezdek, Cluster validity with fuzzy sets, Journal of Cybernetics 3 (3) (1974) 58-73.

[30] U. Maulik, S. Bandyopadhyay, Performance evaluation of some clustering algorithms and validity indices, IEEE Transaction on Pattern Analysis and Machine Intelligence 24 (12) (2002) 1650-1654.

[31] J. Hartigan, Clustering Algorithms, Wiley series in probability and mathematical statistics. Applied Probability and Statistics. New York: Wiley, 1975.

[32] A. Scott, M. Symons, Clustering methods based on likelihood ratio criteria, Biometrics 27 (2) (1971) 387-397.

[33] F. Marriot, Practical problems in a method of cluster analysis, Biometrics 27 (3) (1971) 501-514.

[34] A. Edwards, L. Cavalli-Sforza, A method for cluster analysis, Biometrics 21 (2) (1965) 362-375.

[35] H. Friedman, J. Rubin, On some invariant criteria for grouping data, Journal of the American Statistical Association 62 (320) (1967) 1159-1178.

[36] A. Weingessel, E. Dimitriadou, S. Dolnicar, An examination of indexes for determining the number of clusters in binary data sets, Working Paper No.29 (1999).

[37] J. Cohen, A coefficient of agreement for nominal scales, Educational and Psychological Measurement 20 (1960) 37-46.

[38] J. Jackson, A User's Guide to Principal Components, Wiley series in probability and mathematical statistics. Applied 
Probability and Statistics. New York: Wiley, 1991.

[39] P. Velleman, D. Hoaglin, Applications, Basics and Computing of Exploratory Data Analysis, Boston, Mass.: Duxbury Press, 1981.

[40] T. Etchells, P. Lisboa, Rule extraction from neural networks: a practical and efficient approach, IEEE Transactions on Neural Networks 17 (2) (2006) 374-384.

[41] S. Michiels, S. Koscielny, C. Hill, Prediction of cancer outcome with microarrays: a multiple random validation strategy, The Lancet 365 (9458) (2005) 488-492.

[42] B. Matharoo-Ball, L. Ratcliffe, L. Lancashire, S. Ugurel, A. Miles, D. Weston, R. Rees, D. Schadendorf, G. Ball, C. Creaser, Diagnostic biomarkers differentiating metastatic melanoma patients from healthy controls identified by an integrated MALDI-TOF mass spectrometry/bioinformatic approach, Proteomics Clin. Appl. 1 (6) (2007) 605-620.

[43] R. McClelland, P. Finlay, K. Walker, D. Nicholson, J. Robertson, R. Blamey, R. Nicholson, Automated quantitation of immunocytochemically localized estrogen receptors in human breast cancer, Cancer Res 50 (1990) 3545-3550.

[44] S. Detre, G. Saclani Jotti, M. Dowsett, A "quickscore" method for immunohistochemical semiquantitation: Validation for oestrogen receptor in breast carcinomas, J Clin Pathol 48 (1995) 876-878.

[45] X. Wang, J. Garibaldi, A comparison of fuzzy and non-fuzzy clustering techniques in cancer diagnosis, in: Proceedings of second international conference in Computational Intelligence in Medicine and Healthcare, 2005, pp. 250-256. 


\section{Summary}

In a previous study [12], we have suggested that immunohistochemical analysis may be used to identify distinct biological classes of breast cancer. The objectives of this work were to verify the stability of groups obtained from four different unsupervised clustering algorithms, applied to the same data, and to compare and combine the different solutions with the ones available from the previous study. The clustering techniques were used to divide our dataset in six groups, which were labelled according to our previous classification [12]. Moreover, where appropriate, validity indices were used to explore the best data subdivision and to validate the obtained classification. Despite the fact that fuzzy c-means is one of the most widely used clustering techniques, results obtained from the algorithm were quite poor and were dropped from further investigation. The PAM algorithm produce somewhat different results to the other techniques, so that correspondences between classifications were also difficult. Then, only considering Hierarchical, K-means and ART methods, a set of six core classes was elucidated by a form of consensus clustering in which labels assigned to the groups were aligned to find correspondences among patients grouped in similar clusters by different techniques. It was found that around the $62 \%$ (663 patients) of the available data was assigned to classes, while the remaining 413 women (38\%) presented mixed class characteristics. The use of different clustering methods has, once again, demonstrated that diverse algorithms will in general produce different clusters, particularly when large multi-dimensional data sets are considered. 


\section{List of Figures}

1 Cluster validity indices obtained for K-means and PAM clustering, for varying cluster numbers

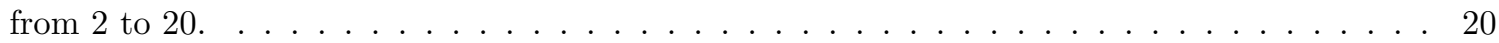

2 Biplots of clusters projected on the first and second principal component axes. . . . . . . . 21

3 Biplots of classes projected on the first and second principal component axes . . . . . . . . 21

4 Boxplot for all markers, whole data and grouped by class . . . . . . . . . . . . . . . . . . 22

5 A summary of the classes of breast cancer obtained, with indicative class interpretations. . . 23

6 Boxplots of Nottingham Prognostic Index (NPI) by class. . . . . . . . . . . . . . . . . . . 23 

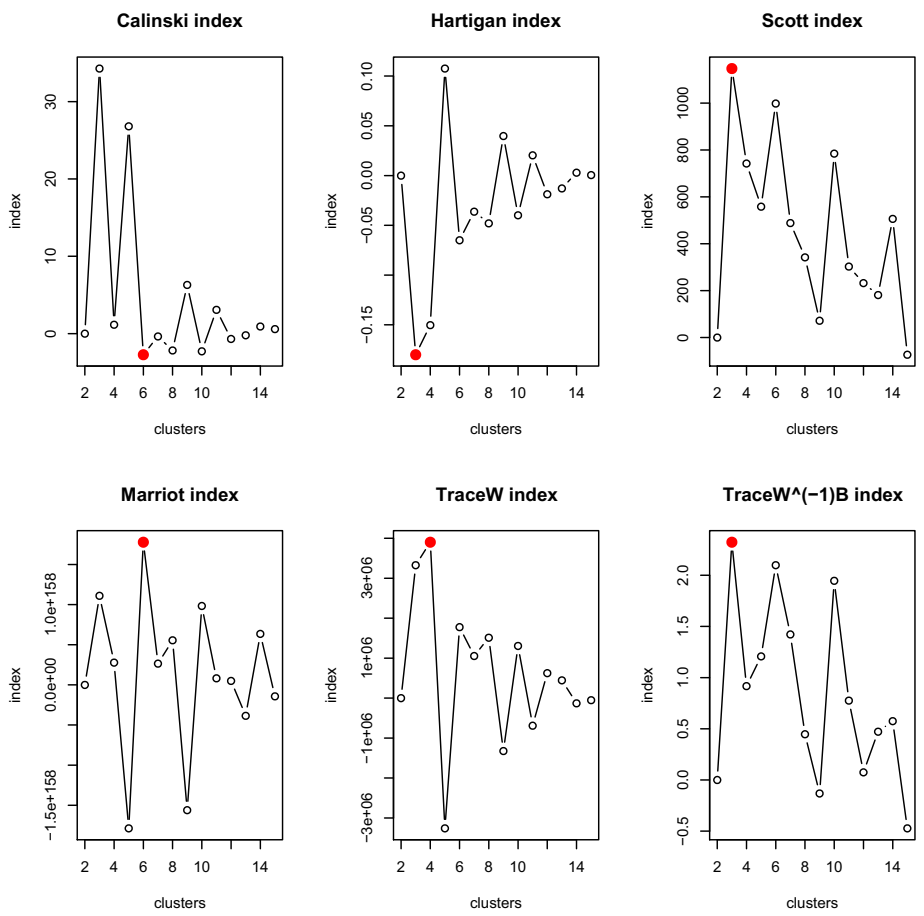

(a) K-means indices behaviors
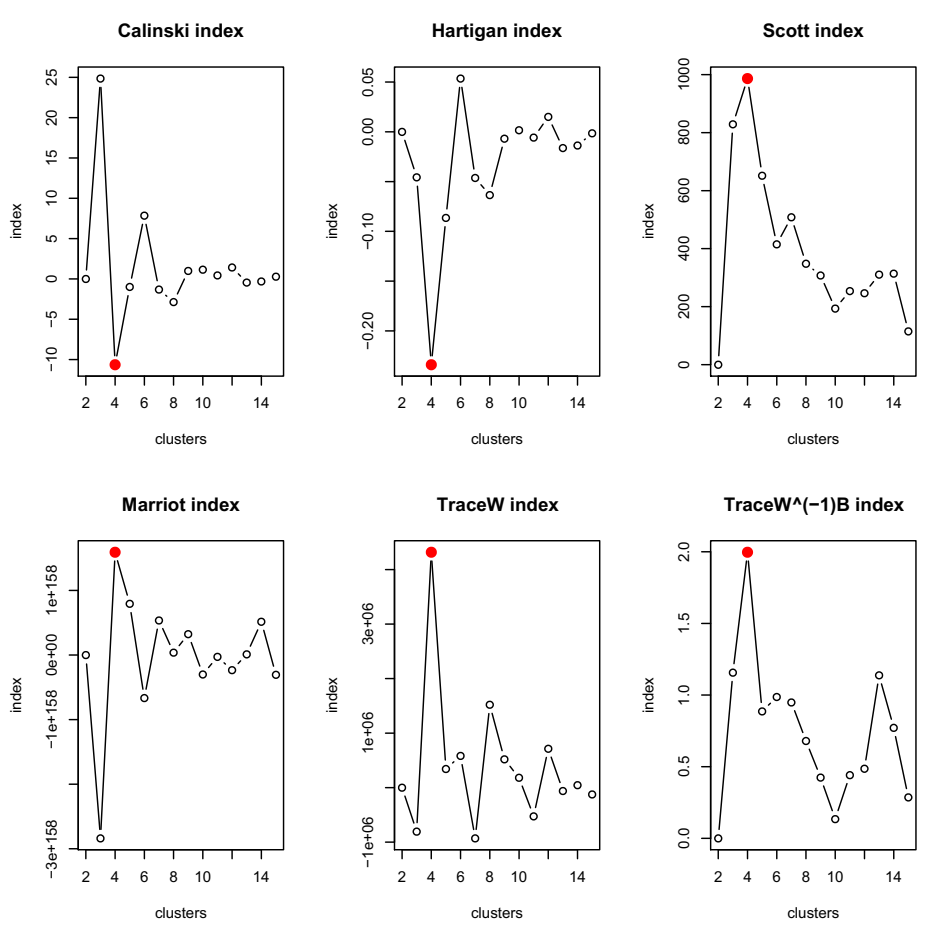

(b) PAM indices behaviors

Figure 1: Cluster validity indices obtained for K-means and PAM clustering, for varying cluster numbers from 2 to 20. 


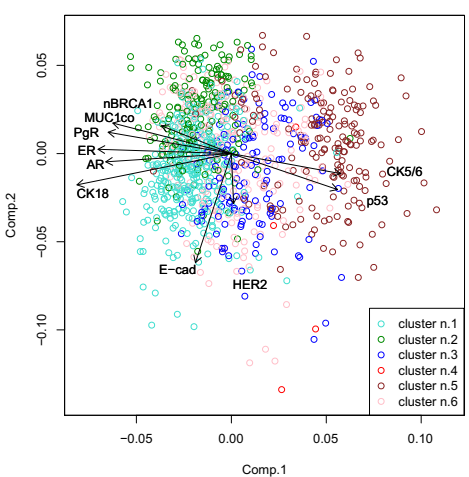

(a) Hierarchical clustering

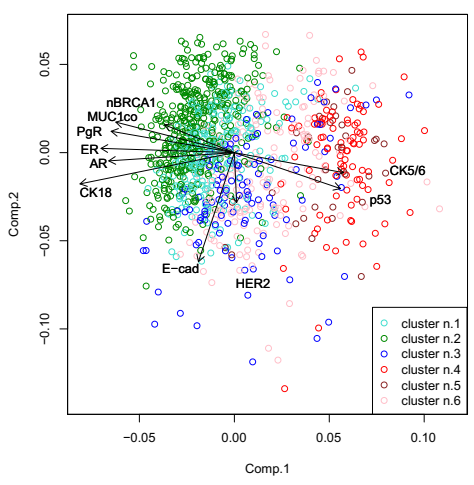

(c) ART clustering

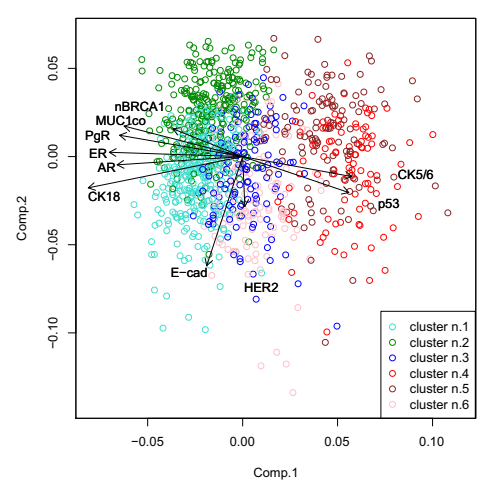

(b) K-means clustering

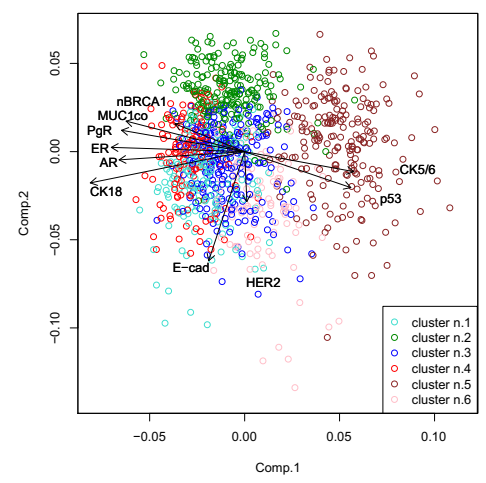

(d) PAM clustering

Figure 2: Biplots of clusters projected on the first and second principal component axes.

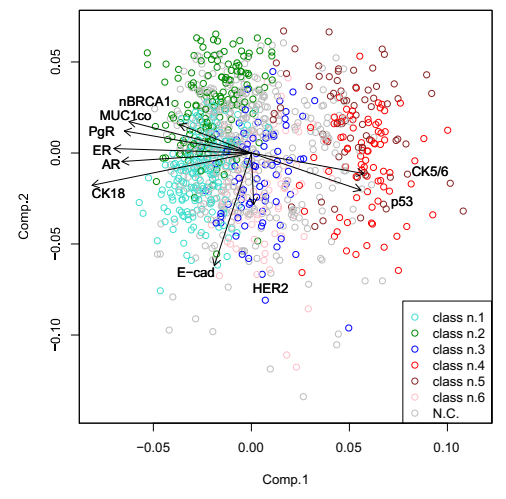

(a) For all patients

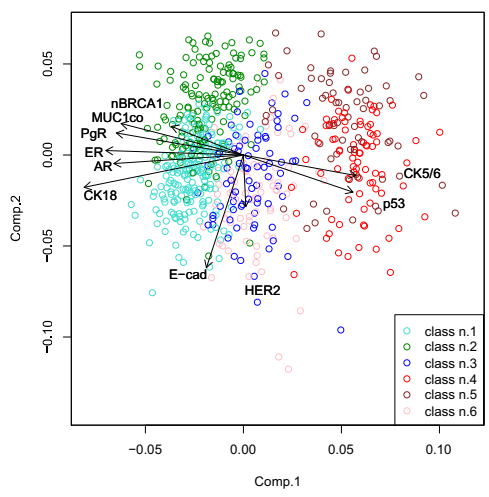

(b) For only patients in classes 1-6

Figure 3: Biplots of classes projected on the first and second principal component axes 


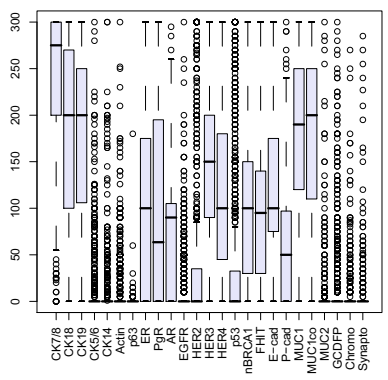

(a) For all patients

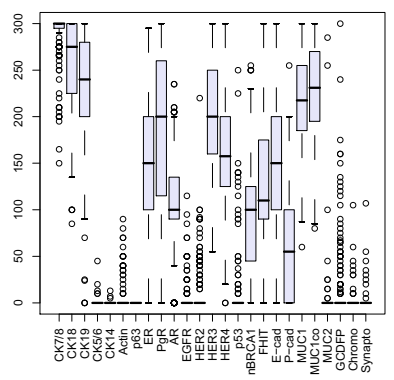

(c) Class 1

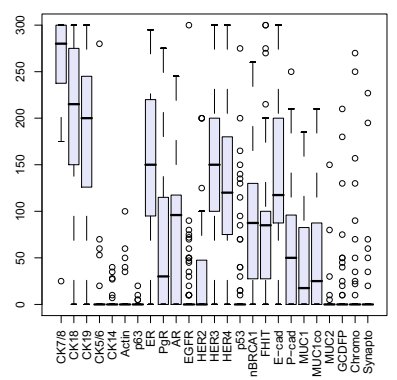

(e) Class 3

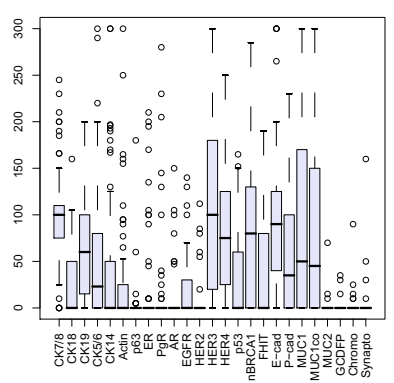

(g) Class 5

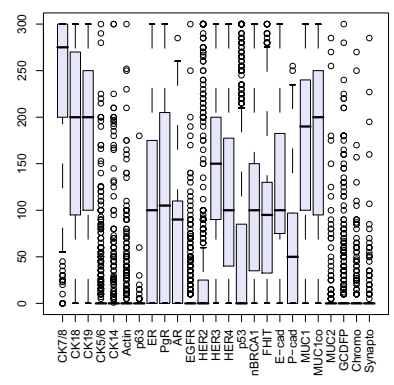

(b) For patients in classes 1-6

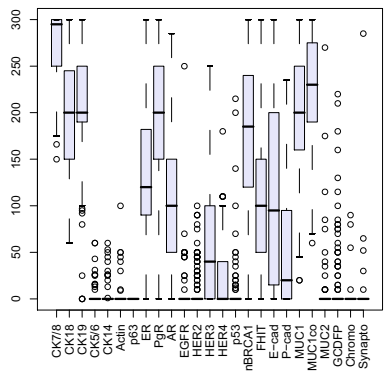

(d) Class 2

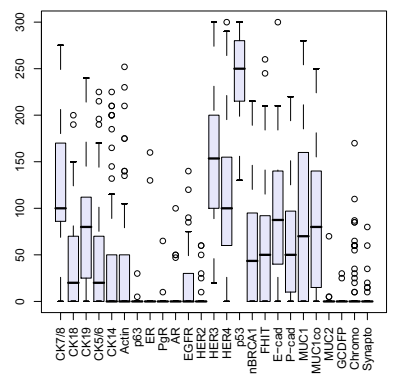

(f) Class 4

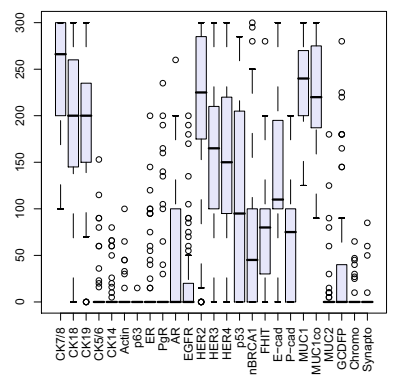

(h) Class 6

Figure 4: Boxplot for all markers, whole data and grouped by class 


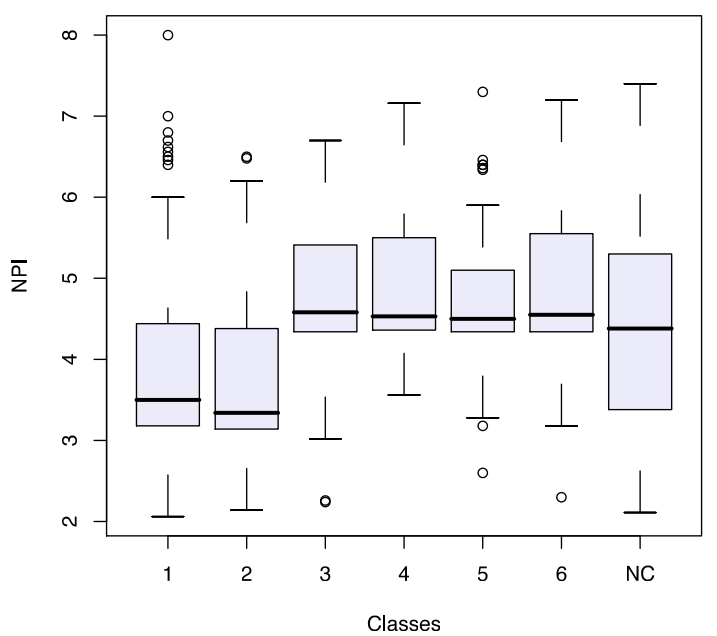

Figure 6: Boxplots of Nottingham Prognostic Index (NPI) by class. 


\section{List of Tables}

1 Different validity indices and their associated decision rules . . . . . . . . . . . . . . . 25

2 Complete list of antibodies used and their dilutions . . . . . . . . . . . . . . . . . 25

3 H-score of each variable for three different patients . . . . . . . . . . . . . . . . 26

4 Kappa and weighted kappa index among different classifications . . . . . . . . . . . . . . . 26

5 Number of cases in each cluster . . . . . . . . . . . . . . . . . . . . . . 26

6 Rules for determining consensus classes . . . . . . . . . . . . . . . . . . . . . 27

7 Description of classes as determined by statistical characterisation. . . . . . . . . . . . . . . 27

8 A summary of rules obtained from the automated methods for defining class memberships . . 27

9 Breast Cancer Class distribution in relation to clinicopathological parameters (NST: No Special Type). . . . . . . . . . . . . . . . . . . . . . . . . . . 28 
Table 1: Different validity indices and their associated decision rules

\begin{tabular}{ll}
\hline Index & Decision rule \\
\hline Calinski and Harabasz & $\min _{n}\left(\left(i_{n+1}-i_{n}\right)-\left(i_{n}-i_{n-1}\right)\right)$ \\
Hartigan & $\min _{n}\left(\left(i_{n+1}-i_{n}\right)-\left(i_{n}-i_{n-1}\right)\right)$ \\
Scott and Symons & $\max _{n}\left(i_{n}-i_{n-1}\right)$ \\
Marriot & $\max _{n}\left(\left(i_{n+1}-i_{n}\right)-\left(i_{n}-i_{n-1}\right)\right)$ \\
Trace $W$ & $\max _{n}\left(\left(i_{n+1}-i_{n}\right)-\left(i_{n}-i_{n-1}\right)\right)$ \\
Trace $W^{-1} B$ & $\max _{n}\left(i_{n}-i_{n-1}\right)$ \\
\hline
\end{tabular}

Table 2: Complete list of antibodies used and their dilutions

\begin{tabular}{|c|c|c|}
\hline Antibody, clone & Short Name & Dilution \\
\hline \multicolumn{3}{|l|}{ Luminal phenotype } \\
\hline CK 7/8 [clone CAM 5.2] & $\mathrm{CK} 7 / 8$ & $1: 2$ \\
\hline CK 18 [clone DC10] & CK18 & $1: 50$ \\
\hline CK 19 [clone BCK 108] & CK19 & $1: 100$ \\
\hline \multicolumn{3}{|l|}{ Basal Phenotype } \\
\hline CK 5/6 [cloneD5/16134] & CK5/6 & $1: 100$ \\
\hline CK 14 [clone LL002] & CK14 & $1: 100$ \\
\hline SMA [clone 1A4] & Actin & $1: 2000$ \\
\hline p63 ab-1 [clone 4A4] & p63 & $1: 200$ \\
\hline \multicolumn{3}{|l|}{ Hormone receptors } \\
\hline ER [clone 1D5] & ER & $1: 80$ \\
\hline PgR [clone PgR 636] & PgR & $1: 100$ \\
\hline AR [clone F39.4.1] & $\mathrm{AR}$ & $1: 30$ \\
\hline \multicolumn{3}{|l|}{ EGFR family members } \\
\hline EGFR [clone EGFR.113] & EGFR & $1: 10$ \\
\hline HER2/c-erbB-2 & HER2 & $1: 250$ \\
\hline HER3/c-erbB-3 [clone RTJ1] & HER3 & $1: 20$ \\
\hline HER4/c-erbB-4 [clone HFR1] & HER4 & $6: 4$ \\
\hline \multicolumn{3}{|l|}{ Tumour suppressor genes } \\
\hline p53 [clone DO7] & p53 & $1: 50$ \\
\hline nBRCA1 Ab-1 [clone MS110] & nBRCA1 & $1: 150$ \\
\hline Anti-FHIT [clone ZR44] & FHIT & $1: 600$ \\
\hline \multicolumn{3}{|l|}{ Cell adhesion molecules } \\
\hline Anti E-cad [clone HECD-1] & E-cad & $1: 10 / 20$ \\
\hline Anti P-cad [clone 56] & P-cad & $1: 200$ \\
\hline \multicolumn{3}{|l|}{ Mucins } \\
\hline NCL-Muc-1 [clone Ma695] & MUC1 & $1: 300$ \\
\hline NCL-Muc-1 core [clone Ma552] & MUC1co & $1: 250$ \\
\hline NCL muc2 [clone Ccp58] & MUC2 & $1: 250$ \\
\hline \multicolumn{3}{|l|}{ Apocrine differentiation } \\
\hline Anti-GCDFP-15 & GCDFP & $1: 30$ \\
\hline \multicolumn{3}{|l|}{ Neuroendocrine differentiation } \\
\hline Chromogranin A [clone DAK-A3] & Chromo & $1: 100$ \\
\hline Synaptophysin [clone SY38] & Synapto & $1: 30$ \\
\hline
\end{tabular}


Table 3: H-score of each variable for three different patients

\begin{tabular}{lccc}
\hline Variable name & Patient 1 & Patient 300 & Patient 1061 \\
\hline CK7/8 & 200 & 280 & 100 \\
CK18 & 300 & 190 & 0 \\
CK19 & 200 & 145 & 0 \\
CK5/6 & 0 & 0 & 125 \\
CK14 & 0 & 0 & 210 \\
Actin & 0 & 0 & 0 \\
p63 & 0 & 0 & 0 \\
ER & 60 & 110 & 0 \\
PgR & 0 & 0 & 0 \\
AR & 0 & 45 & 0 \\
EGFR & 0 & 0 & 0 \\
HER2 & 300 & 0 & 0 \\
HER3 & 170 & 72 & 250 \\
HER4 & 100 & 130 & 150 \\
p53 & 150 & 0 & 270 \\
nBRCA1 & 200 & 45 & 0 \\
FHIT & 120 & 0 & 0 \\
E-cad & 100 & 115 & 0 \\
P-cad & 0 & 15 & 0 \\
MUC1 & 180 & 255 & 160 \\
MUC1co & 210 & 210 & 80 \\
MUC2 & 0 & 0 & 0 \\
GCDFP & 90 & 0 & 0 \\
Chromo & 0 & 0 & 0 \\
Synapto & 0 & 0 & 0 \\
\hline & & &
\end{tabular}

Table 4: Kappa and weighted kappa index among different classifications

\begin{tabular}{lccc} 
& K-means & ART & PAM \\
\hline HCA & 0.497 & 0.296 & 0.325 \\
& 0.548 & 0.401 & 0.332 \\
K-means & - & 0.494 & 0.420 \\
& - & 0.599 & 0.525 \\
ART & - & - & 0.224 \\
& - & - & 0.376 \\
\hline
\end{tabular}

Table 5: Number of cases in each cluster

\begin{tabular}{lrrr}
\hline Cluster & HCA & K-means & ART \\
\hline 1 & 336 & 301 & 238 \\
2 & 180 & 282 & 408 \\
3 & 139 & 138 & 111 \\
4 & 4 & 97 & 96 \\
5 & 183 & 124 & 35 \\
6 & 234 & 134 & 188 \\
\hline
\end{tabular}


Table 6: Rules for determining consensus classes

\begin{tabular}{llr}
\hline If cluster . . & Class & No. of cases \\
\hline H1 \& KM1 \& (ART1 | ART2) & 1 & 202 \\
H2 \& KM2 \& (ART1 | ART2) & 2 & 153 \\
H3 \& KM3 & 3 & 80 \\
H5 \& KM4 \& ART4 & 4 & 82 \\
H5 \& KM5 & 5 & 69 \\
H6 \& KM6 \& ART6 & 6 & 77 \\
\hline \multicolumn{2}{l}{ Total number of cases assigned to classes 1-6 } & 663 \\
\multicolumn{2}{l}{ Total number of cases not classified } & 413 \\
\hline
\end{tabular}

Table 7: Description of classes as determined by statistical characterisation.

\begin{tabular}{llll}
\hline Class & Over-expressed & Under-expressed & Other \\
\hline 1 & ER, AR, PgR, HER3, HER4 & & \\
2 & ER, AR, PgR, nBRCA1 & HER3, HER4 & PgR normal \\
3 & ER, AR & MUC1, MUC1co & \\
4 & p53 & ER, PgR, HER2, MUC1, MUC1co, & \\
5 & & EK18, CK7/8, CK19 & \\
& & CK18, CK7/8, CK19 1, MUC1co, & p53 absent \\
6 & HER2, HER3, HER4 & & ER, AR, PgR absent; \\
& & & p53 widely spread \\
\hline
\end{tabular}

Table 8: A summary of rules obtained from the automated methods for defining class memberships

\begin{tabular}{|c|c|c|}
\hline Class & Over-expressed & Under-expressed \\
\hline $1(\mathrm{ANN})$ & PgR, HER3, HER4, MUC1co & \\
\hline 1 (OSRE) & PgR, HER3, HER4, CK18, CK19, MUC1co & HER2 \\
\hline $2(\mathrm{ANN})$ & PgR, nBRCA1 & HER3, HER4 \\
\hline 2 (OSRE) & PgR, nBRCA1, MUC1co & HER3, HER4 \\
\hline $3(\mathrm{ANN})$ & ER & MUC1 \\
\hline 3 (OSRE) & CK7/8, CK18 & \\
\hline $4(\mathrm{ANN})$ & p53 & \\
\hline 4 (OSRE) & HER3, p53 & ER, HER2 \\
\hline $5(\mathrm{ANN})$ & CK5 $/ 6$ & CK7/8 \\
\hline 5 (OSRE) & & p53; CK7/8, CK19 or HER2, HER4 \\
\hline $6(\mathrm{ANN})$ & HER2 & \\
\hline 6 (OSRE) & HER2, p53, MUC1co & ER \\
\hline
\end{tabular}


Table 9: Breast Cancer Class distribution in relation to clinicopathological parameters (NST: No Special Type).

\begin{tabular}{|c|c|c|c|c|c|c|c|}
\hline & \multicolumn{6}{|c|}{ Breast Cancer Class } & \multirow[b]{2}{*}{$\phi$} \\
\hline & 1 & 2 & 3 & 4 & 5 & 6 & \\
\hline \multicolumn{8}{|l|}{ Age } \\
\hline$\leq 50$ & $76(37.6)$ & $63(41.2)$ & $24(30.0)$ & $55(67.1)$ & $33(47.8)$ & $37(48.1)$ & \multirow{3}{*}{0.209} \\
\hline$>50$ & $126(62.4)$ & $90(58.8)$ & $56(70.0)$ & $27(32.9)$ & $36(52.2)$ & $40(51.9)$ & \\
\hline Total & 202 & 153 & 80 & 82 & 69 & 77 & \\
\hline \multicolumn{8}{|l|}{ Grade } \\
\hline 1 & $58(28.9)$ & $43(28.1)$ & $2(2.5)$ & $0(0)$ & $2(2.9)$ & $1(1.3)$ & \multirow{4}{*}{0.660} \\
\hline 2 & $81(40.2)$ & $89(58.2)$ & $18(22.5)$ & $1(1.2)$ & $7(10.1)$ & $12(15.6)$ & \\
\hline 3 & $62(30.8)$ & $21(13.7)$ & $60(75.0)$ & $81(98.8)$ & $60(87.0)$ & $64(83.1)$ & \\
\hline Total & 201 & 153 & 80 & 82 & 69 & 77 & \\
\hline \multicolumn{8}{|l|}{ Size } \\
\hline$\leq 1.5 \mathrm{~cm}$ & $79(39.1)$ & $65(42.5)$ & $20(25.0)$ & $12(14.6)$ & $15(21.7)$ & $16(20.8)$ & \multirow[t]{3}{*}{0.225} \\
\hline$>1.5 \mathrm{~cm}$ & $123(60.9)$ & $88(57.5)$ & $60(75.0)$ & $70(85.4)$ & $54(78.3)$ & $61(79.2)$ & \\
\hline Total & 202 & 153 & 80 & 82 & 69 & 77 & \\
\hline \multicolumn{8}{|l|}{ Lymph Node Stage } \\
\hline 1 & $132(65.3)$ & $108(70.6)$ & $39(48.7)$ & $50(61.0)$ & $52(75.4)$ & $36(46.8)$ & \multirow{4}{*}{0.217} \\
\hline 2 & $58(28.7)$ & $37(24.2)$ & $35(43.8)$ & $23(28.0)$ & $10(14.5)$ & $30(39.0)$ & \\
\hline 3 & $12(5.9)$ & $7(4.6)$ & $6(7.5)$ & $9(11.0)$ & $7(10.1)$ & $10(13.0)$ & \\
\hline Total & 202 & 152 & 80 & 82 & 69 & 76 & \\
\hline \multicolumn{8}{|l|}{ Tumour type } \\
\hline Invasive ductal/NST & $97(48.0)$ & $45(29.4)$ & $64(80.0)$ & $70(85.4)$ & $53(76.8)$ & $68(88.3)$ & \multirow{9}{*}{0.622} \\
\hline Tubular mixed & $52(25.7)$ & $50(32.6)$ & $8(10.0)$ & $0(0)$ & $1(1.5)$ & $5(6.5)$ & \\
\hline Medullary & $0(0)$ & $0(0)$ & $0(0)$ & $10(12.2)$ & $5(7.2)$ & $2(2.6)$ & \\
\hline Lobular & $18(8.9)$ & $34(22.2)$ & $6(7.5)$ & $0(0)$ & $4(5.8)$ & $1(1.3)$ & \\
\hline Special types & $19(9.4)$ & $11(7.2)$ & $0(0)$ & $1(1.2)$ & $0(0)$ & $0(0)$ & \\
\hline Mixed NST \& lobular & $6(3.0)$ & $7(4.6)$ & $2(2.5)$ & $1(1.2)$ & $3(4.3)$ & $0(0)$ & \\
\hline Mixed NST \& special type & $9(4.5)$ & $5(3.3)$ & $0(0)$ & $0(0)$ & $1(1.5)$ & $0(0)$ & \\
\hline Miscellaneous & $0(0)$ & $1(0.7)$ & $0(0)$ & $0(0)$ & $2(2.9)$ & $0(0)$ & \\
\hline Total & 201 & 153 & 80 & 82 & 69 & 76 & \\
\hline
\end{tabular}


None Declared.

\section{Conflict of Interest Statement}

\section{Conflict of Interest Statement}

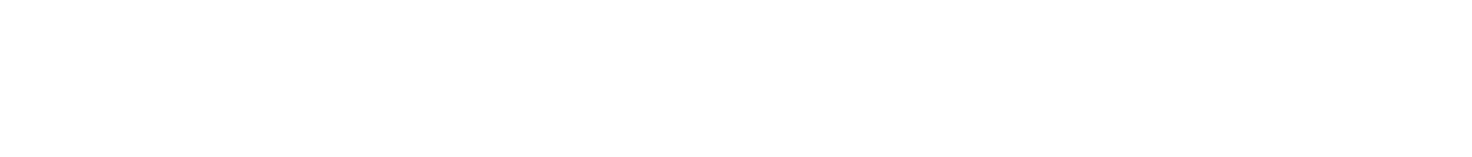

(1)

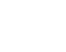

a

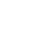

(1)
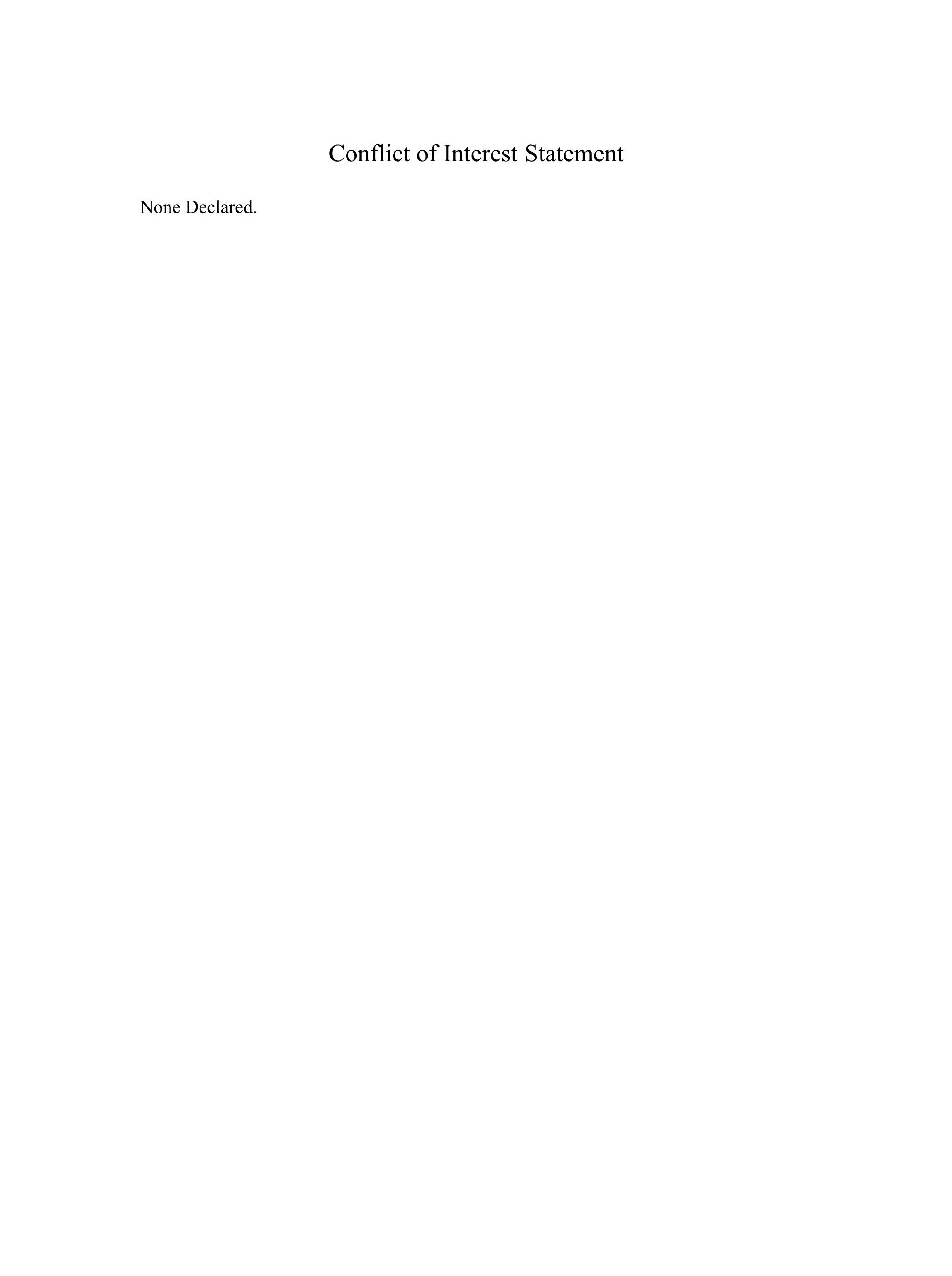